\title{
Commensurability Effects for Fermionic Atoms Trapped in 1D Optical Lattices
}

\author{
Rafael A. Molina, ${ }^{1}$ Jorge Dukelsky, ${ }^{1}$ and Peter Schmitteckert ${ }^{2}$ \\ ${ }^{1}$ Instituto de Estructura de la Materia-CSIC, Serrano 123, 28006 Madrid, Spain \\ ${ }^{2}$ Institut für Theorie der Kondensierten Materie, Universität Karlsruhe, 76128 Karlsruhe, Germany \\ (Received 29 March 2007; revised manuscript received 21 June 2007; published 24 August 2007)
}

Fermionic atoms in two different hyperfine states confined in optical lattices show strong commensurability effects due to the interplay between the atomic density wave ordering and the lattice potential. We show that spatially separated regions of commensurable and incommensurable phases can coexist. The commensurability between the harmonic trap and the lattice sites can be used to control the amplitude of the atomic density waves in the central region of the trap.

DOI: 10.1103/PhysRevLett.99.080404

PACS numbers: 05.30.Fk, 03.75.Ss, 71.10.Pm

Ultracold Bose or Fermi gases can be confined in artificial optical lattices created by standing-wave laser fields. The low-energy properties of these systems can be described using models borrowed from condensed matter systems [1] whose parameters and dimensionality can be controlled with high precision. This tunability has opened new avenues for understanding the physics of strongly correlated systems. Greiner et al. observed a superfluid to Mott insulator transition in a 3D optical lattice with bosonic ${ }^{87} \mathrm{Rb}$ atoms [2]. Interesting experimental results have also been obtained for fermions [3].

The physics of cold fermionic atoms in optical lattices is predicted to be the one of the attractive Hubbard model with the different hyperfine states playing the role of the spin states. In the case of one dimension, bosonization predicts the formation of a Luther-Emery liquid for attractive interactions [4]. The spin sector is gapped, inducing exponential decay of spin correlations in contrast to singlet superconducting and charge-density wave correlations that have a power law decay [5]. Atoms are trapped in experiments and the interplay between correlations, lattice, and confinement has to be properly addressed. Moreover, the study of the inhomogeneity can be very relevant for the understanding of unconventional superconductors. For example, the density of states of high- $T_{c}$ superconductors presents spatial inhomogeneities [6]. Confined fermions in optical lattices are ideal experimental candidates to study these issues.

Recently, Gao Xianlong et al. studied the unpolarized attractive Hubbard model in a 1D optical lattice with harmonic confinement. Using the density matrix renormalization group (DMRG) approach, they observed the coexistence of spin pairing with an atomic density wave (ADW) that could be interpreted as a signature of a Luther-Emery liquid phase. This interpretation is further justified by the fact that the momentum of the ADW is proportional to the average atomic density in the bulk of the trap $k_{\mathrm{ADW}}=\pi \bar{n}$. These ADWs could be detected measuring the elastic lightscattering diffraction pattern (the Fraunhofer structure factor), proportional to the Fourier transform of the atomic density [7]. In a complementary work using quantum Monte Carlo simulations, Pour et al. interpreted the divergence of the form factors of the density-density and pairing correlation function as defining a supersolid [8].

The purpose of this Letter is to uncover new commensurability effects induced by the combination of the lattice and confinement potentials and the atomic density. Since the precise momentum of the ADWs in the trap can be controlled with the average density, it is possible to observe effects similar to the commensurabilityincommensurability transition appearing in crystalline surfaces when the density oscillations have a different periodicity than the underlying lattice [9]. These concepts have also been applied to the doped Mott transition in strongly correlated Fermi systems $[5,10]$. We will show that for local densities close to half filling there appear commensurate and incommensurate phases in different parts of the lattice. Similar local quantum criticality issues have been seen before in the repulsive case [11,12]. In the commensurate phase that arises in sectors of the lattice where the density is close to half filling, the amplitude of the ADWs is enhanced due to local umklapp scattering terms. The incommensurate phase close to half filling is characterized by the appearance of a new length scale, similar to a beating length, due to the interplay between the periodicity of the lattice and the periodicity of the ADW that results in nodes and amplitude modulation of the latter.

Similar to previous works, we consider the Hamiltonian

$$
\begin{aligned}
\hat{H}= & -t \sum_{i, \sigma}\left(\hat{c}_{i \sigma} \hat{c}_{i+1 \sigma}+\text { H.c. }\right)+U \sum_{i} \hat{n}_{i \uparrow} \hat{n}_{i \downarrow} \\
& +V \sum_{i}(i-L / 2+D)^{2} \hat{n}_{i},
\end{aligned}
$$

where $t$ is the hopping, $\sigma$ is a pseudospin- $1 / 2$ degree of freedom, $U$ is the interaction (always attractive $U<0$ ), and $V$ is the strength of the confinement. The creation, destruction, and number operators are the usual ones at each site $i$ of the lattice. The total length $L$ of the system is chosen such that the density is smooth, going to zero in the edges. All energies are expressed in units of $t(t=1)$. The 
parameter $D$ measures the displacement of the center of the harmonic confinement potential with respect to the position of a lattice site. It can vary from 0.0 (the center of the harmonic well coincides with a lattice site) to 0.5 (the center of the well is exactly between two lattice sites). We will study only the unpolarized case.

We study the ground state properties of the Hamiltonian (1) with the DMRG algorithm [13] that provides very accurate numerical results. Because of the breaking of the translational symmetry by the trapping potential some modifications of the DMRG procedure are required. Here we follow the same procedure used before for disorder potentials [14] and umklapp scattering induced phase transitions [15]. In order to obtain enough accuracy for the largest size systems we needed to keep a maximum of 1400 states in each iteration.

As a complementary tool we use the approximate Hartree-Fock (HF) method. It will allow us to study system sizes significantly beyond the limits of a DMRG calculation. The HF approximation describes with high quantitative precision the ADWs for $|U|<1$ as we have checked for systems up to $L=250$ by comparison with DMRG results. For values of $U$ between $U=-1$ and $U=-2$ the $\mathrm{HF}$ description is qualitatively good for the ADWs but it overestimates their amplitude. For $|U|>2 \mathrm{HF}$ deviates from the exact results due to strong pairing correlations not included in the approximation.

Without the trapping potential bosonization predicts an $\mathrm{ADW}$ with wave number $k_{\mathrm{ADW}}=2 k_{F}$ [5]. Adding confinement, the wave number of the ADW is modulated by the density profile in the trap, but it can still be written as a function of an effective Fermi wave number $k_{F}^{\text {eff }}$ [7]

$$
k_{\mathrm{ADW}}=2 k_{F}^{\mathrm{eff}}, \quad k_{F}^{\mathrm{eff}}=\pi \bar{n} / 2,
$$

where $\bar{n}$ is the average density around the center of the trap. For $\bar{n}=1.0$ (half filling) $k_{\mathrm{ADW}}=\pi$ and we have an ADW with a periodicity of two lattice sites.

Figure 1 shows DMRG results for the site density of a system with $L=180, U=-1, V=0.0005, D=0.0$ and two values of the number of atoms $N=N_{\uparrow}+N_{\downarrow}$. $N=80$ is represented by open squares and $N=90$ by open circles. We also show for comparison HF results for the same system. The agreement between DMRG and HF is remarkable for $U=-1$. As can be seen in the figure, the local density is close to half filling in the center of the trap. As a consequence, an ADW with a long range modulation of the amplitudes develops. The effect is more pronounced in the case with $N=90$, displaying a nice commensurate ADW with large amplitude. The density in the center of the trap is below half filling for $N=80$; however, the ADW can still be seen.

The appearance of lobes and nodes in the ADW depends on small deviations of $k_{\mathrm{ADW}}$ from the lattice momentum $\pi$. These features are expected to be more pronounced for larger lattices. Therefore, we resort to the HF approximation to treat lattice sizes that are not attainable with present

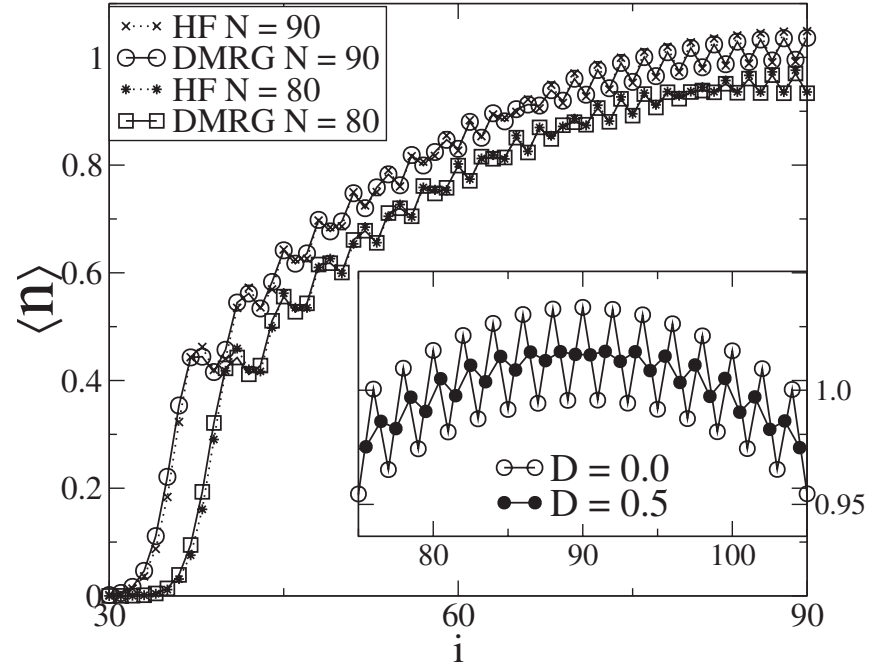

FIG. 1. DMRG and HF results for $L=180, V=0.0005, D=$ $0.0, U=-1.0$, and $N=80,90$. As the graph is symmetric, only the left part is shown. The inset shows a zoom of the central region for $N=90$ comparing DMRG results with $D=0.0$ and $D=0.5$.

DMRG codes. In order to relate intermediate and large lattice systems we use the scaling $N V^{2}=$ constant while $N \rightarrow \infty$ and $V \rightarrow 0$ [16]. This scaling keeps constant the density in the central region of the trap. In Fig. 2 we show HF results for the density in the central region for systems with $L=1000, V=0.000012, U=-1$ and different number of atoms, to illustrate the kind of ADWs appearing in larger size systems as a function of the number of atoms. Of particular interest is the case of $N=560$ where we can see the suppression of the ADW for a displacement $D=$ 0.5 of the trap.

The distance between nodes $\delta$ in the ADW is related to the distance it takes for the system to realize it is not

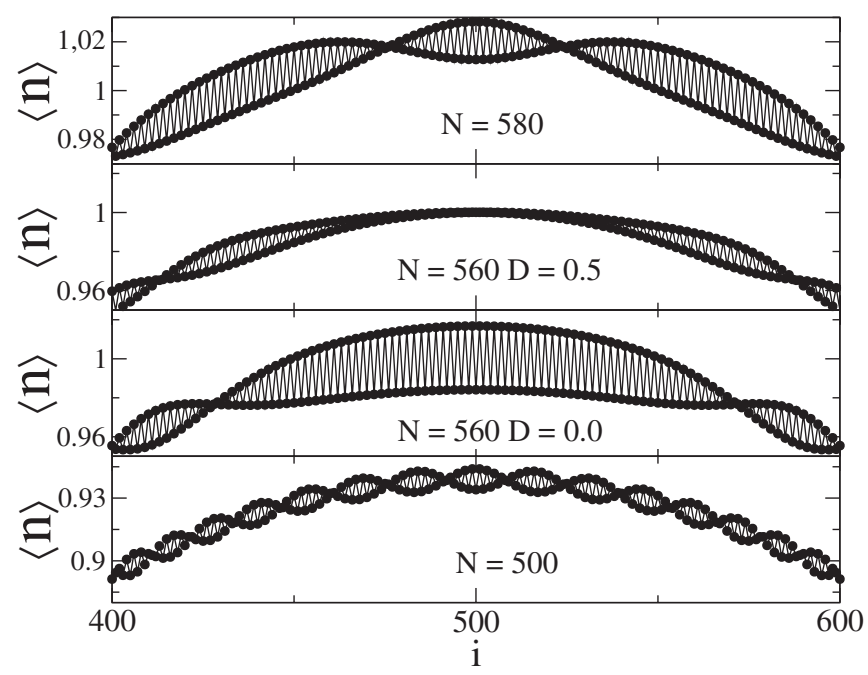

FIG. 2. HF local density as a function of the site index for a system of $L=1000$ sites and different values of $N$ and $D$. 
commensurate with the underlying lattice. If the local average density is close to half filling $\bar{n}=1 \pm \epsilon$, we have from (2) $k_{\mathrm{ADW}}=\pi(1 \pm \epsilon) . \delta$ can be from the distance between nodes for a wave $\cos [\pi(1 \pm \epsilon) x]$ taken at integer values of the variable $x$, leading to $\delta=\epsilon^{-1}$.

Figure 3 shows HF and DMRG results of the distance between nodes $\delta$ versus the average local density in the center of the trap. The continuous line represents the formula $\delta=\epsilon^{-1}$. Because of finite size effects, slight deviations could be seen close to half filling. However, the overall agreement is excellent.

The enhancement of the ADW amplitude close to half filling can be explained in terms of the additional umklapp interaction. In the bosonization language these effects are treated by adding a term to the umklapp part of the Hamiltonian [5]

$$
H_{\delta}=g_{3} \int d x \cos [\sqrt{8} \phi(x)-\delta x],
$$

where $g_{3}$ is the coupling constant of the umklapp scattering terms, proportional to $U$ in our model, and $\phi(x)$ is the bosonized field. The additional lowering of the energy due to the umklapp term is effective even for local densities not precisely at half filling. This phenomenon is robust close to half filling, giving rise to the enhancement of the amplitude and range modulation of the ADW. The amplitude of the ADW increases with the value of the attractive interaction as shown in the inset of Fig. 3 for DMRG and HF calculations with $L=100$ and $V=0.001$ but changing the number of particles for each value of the interaction to keep local half filling in the trap center. The increase is very abrupt for small values of $|U|$, saturating for $|U| \sim 10$.

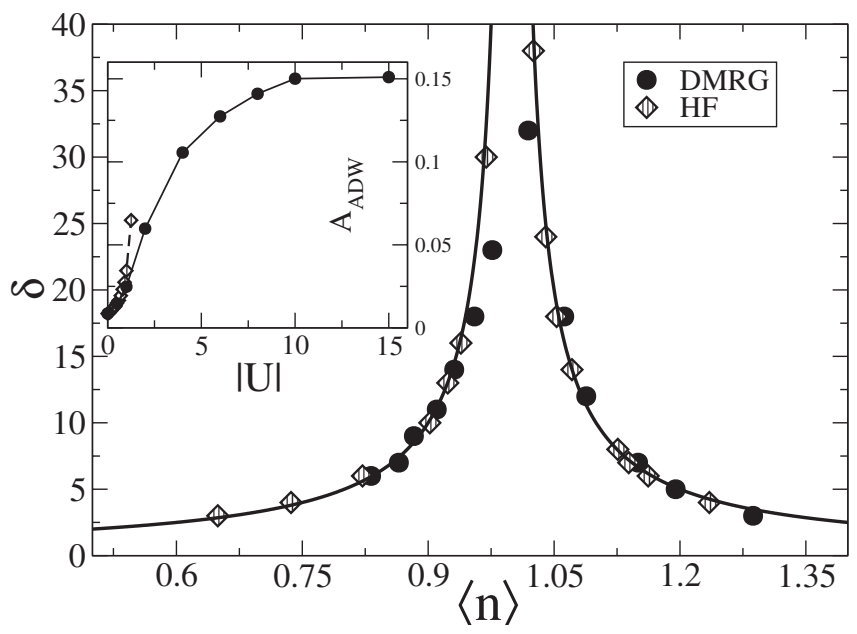

FIG. 3. Distance between nodes $(\delta)$ as a function of the average value of the density in the center of the trap for HF calculations with $L=1000, V=0.000012$, and $U=-1.0$, and DMRG results with $L=100, V=0.001$, and $U=-4$.0. Inset: amplitude of the ADW $A_{\mathrm{ADW}}$ in the center of the trap for different values of the interaction strength with the central density at half filling, $L=100$, and $V=0.001$. DMRG results (๑), HF results for interaction strengths up to $U=-1.5(\diamond)$.
Another important source of commensurability comes from the external trapping potential. Just at half filling a phase difference in the ADW can have dramatic effects in the observed amplitudes at the lattices sites. The central part of the ADW for $N=90$ is shown in the inset of Fig. 1, comparing the results with $D=0.0$ and $D=0.5$. The strong suppression of the ADW can be clearly seen. Even though the properties of the Luther-Emery liquid are not modified by the displacement of the external potential, the actual position of the lattice sites determine the amplitudes of the ADW. This property was not seen previously in the literature [17], because the systems studied were too small to notice the difference but can have big experimental consequences for the observation of the ADWs. Figure 2 also shows these differences between $D=0.0$ and $D=$ 0.5 for a larger system.

To proceed further with the characterization of the different local phases and commensurability effects we will make use of two local quantities, the local variance of the density $\kappa_{i}=\left\langle n_{i}^{2}\right\rangle-\left\langle n_{i}\right\rangle^{2}$ and the order parameter $O_{\mathrm{CADW}}(i)$ for a local commensurate (pinned) ADW defined as:

$$
O_{\mathrm{CADW}}(i)=\left|\left\langle n_{i}-\frac{1}{2}\left(n_{i+1}+n_{i-1}\right)\right\rangle\right| .
$$

While the local variance $\kappa_{i}$ measures the fraction of spinpaired particles in site $i$, the commensurate ADW order parameter $O_{\mathrm{CADW}}(i)$ measures the local amplitude of the ADW between site $i$ and its near neighbors.

In Fig. 4 we show DMRG results for $L=180, V=$ $0.0005, U=-4, N=70$, and $N=86$. The amplitude of the ADW is much higher than in the examples with $U=$ -1 . The density in the case with $N=70$ is very close to half filling in the center and the amplitude of the ADW very large there. The local umklapp scattering term effectively increases the attraction between fermions close to half filling. The other case has densities larger than $n=1.0$ in the central region. Nodes in the density due to the

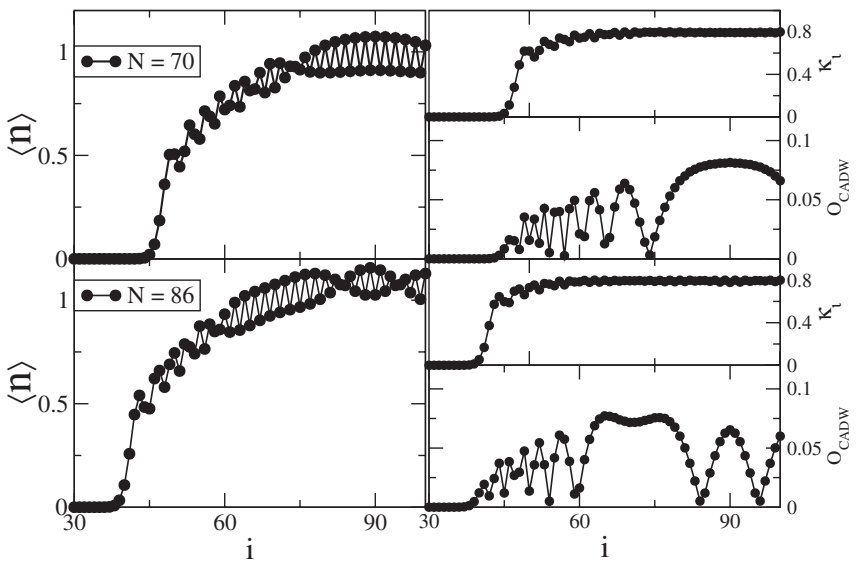

FIG. 4. DMRG results for $L=180, D=0.0, V=0.0005$, and $U=-4$. Top panel: 86 , atoms. Bottom panel: $N=70$. The left panels show the density and the right panels show the local order parameters explained in the text. 


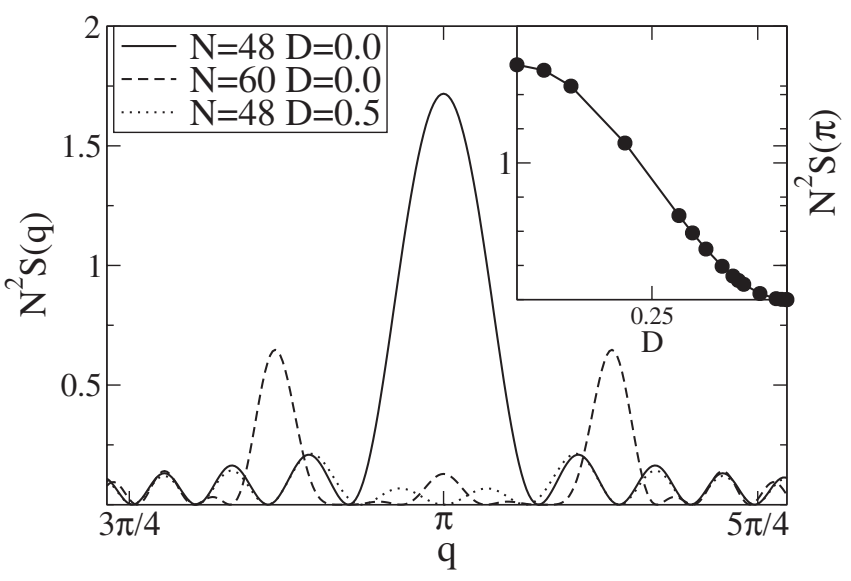

FIG. 5. Structure factor $S(q)$ for different DMRG examples with $L=100$ and $V=0.001$. In the inset we show the value of the height of the peak at $q=\pi$ as a function of $D$ for the example with $N=48$.

incommensurate ADWs appear. The amplitude of the ADWs is larger in the lobe with local density close to half filling and so it is the distance between nodes there. In the right panels we show the value of $\kappa_{i}$ and $O_{\mathrm{CADW}}(i)$ for both examples. The results are close to a plateau in the central region with a large $\kappa_{i}=0.8$. A more careful examination reveals that the maximum of $\kappa_{i}$ occurs in the regions close to half filling, precisely where $O_{\mathrm{CADW}}(i)$ has a maximum.

Lobes and nodes in the ADWs can be observed through elastic light-scattering diffraction experiments, by means of the Fraunhofer structure factor [4]:

$$
S(q)=\frac{1}{N^{2}}\left|\sum_{j} \exp (-i q j) n_{j}\right|^{2} .
$$

Figure 5 shows the structure factor for $L=100, V=$ 0.001 , and $U=-4$. We show three cases with $N=60$ $D=0.0, N=48 D=0.0$, and $N=48 D=0.5$. The largest peak at $q=\pi$ corresponds to the case $N=48$ and $D=0.0$, exactly at half filling. This is due to the large amplitude commensurate ADW. For the same number of particles and $D=0.5$, the ADW disappears and $S(q)$ is suppressed in the region around $q=\pi$. Above half filling ( $N=60)$ a small peak shows up at $q=\pi$, while most of the intensity concentrates at the peaks $q_{\max }=\pi \bar{n}$ and the corresponding symmetric peak. In the inset we show the value of $S(\pi)$ as a function of $D$ to demonstrate that the displacement of the trap is an observable effect.

In conclusion, we have shown that commensurate and incommensurate phases can be seen in cold fermions trapped in optical lattices with attractive interaction. The two phases can appear spatially separated depending on the density and confinement of the atoms in the lattice. The amplitude of the ADW increases with the strength of the interaction. The local density variance $\kappa_{i}$ shows that the commensurate phase is characterized by a maximum number of local spin-paired atoms. The structure factor studied in different examples reveals that the commensurate phase is characterized by a large peak exactly at $k=\pi$, while for phase coexistence the intensity is distributed in several peaks. The displacement of the trap in relation to the lattice is also a very important parameter for the experiment. Depending on it, the amplitude of the ADWs and the $q=$ $\pi$ peak can be suppressed. The width of the peaks is inversely proportional to the number of wells in the optical lattice. This parameter is the main limitation for the experimental detection of this commensurability effects. To resolve the two peaks appearing due to the incommensurability of ADWs with densities $\bar{n}=1.0 \pm \epsilon$ one needs widths of the peaks smaller than $\Gamma<\pi \epsilon$. According to our numerical simulations, for harmonic confinement, the intrinsic width of the peak scales with size as $\Gamma_{\text {int }} \sim 10 / L$. Taking into account that the width induced by the experimental setup, $\Gamma_{\text {exp }}$, will convolute with the intrinsic width, we shall need a system size of $L \gtrsim 10 / \sqrt{4 \epsilon^{2} / \pi^{2}-\Gamma_{\exp }^{2}}$ to resolve the two peaks. Currently available onedimensional optical lattices of 100 sites should be enough to detect the results represented in Fig. 5 if the experimental width is less than 0.24 in units of the density [3].

The authors acknowledge discussions with $\mathrm{M}$. Rodriguez, D. Weinmann, R. M. Noack, S. Rombouts, A. Relaño, and J. González Carmona. This work is supported in part by Spanish Government Grant Ref. FIS2006-12783C03-01 and by Grant CAM-CSIC Ref. 200650M012. R. A. M.'s contract is financed by CSIC and the European Commission through the I3p program.

[1] J. I. Cirac and P. Zoller, Science 301, 176 (2003).

[2] M. Greiner et al., Nature (London) 415, 39 (2002).

[3] T. Stöferle et al., Phys. Rev. Lett. 96, 030401 (2006); J. K. Chin et al., Nature (London) 443, 961 (2006).

[4] A. Luther and V. J. Emery, Phys. Rev. Lett. 33, 589 (1974).

[5] T. Giamarchi, Quantum Physics in One Dimension (Clarendon, Oxford, 2004).

[6] T. Hanaguri et al., Nature (London) 430, 1001 (2004).

[7] Gao Xianlong et al., Phys. Rev. Lett. 98, 030404 (2007).

[8] F. Karim Pour et al., Phys. Rev. B 75, 161104(R) (2007).

[9] V. L. Pokrovsky and A. Talapov, Phys. Rev. Lett. 42, 65 (1979).

[10] T. Giamarchi, Phys. Rev. B 44, 2905 (1991).

[11] M. Rigol, A. Muramatsu, G. G. Batrouni, and R.T. Scalettar, Phys. Rev. Lett. 91, 130403 (2003).

[12] S. Wessel et al., Phys. Rev. A 70, 053615 (2004).

[13] S. R. White, Phys. Rev. Lett. 69, 2863 (1992).

[14] P. Schmitteckert, Ph.D. thesis, Universität Augsburg, 1996; P. Schmitteckert, in Lecture Notes in Physics, edited by I. Peschel, X. Wang, M. Kaulke, and K. Hallberg (Springer, Berlin, 1999), Vol. 528.

[15] P. Schmitteckert and R. Werner, Phys. Rev. B 69, 195115 (2004).

[16] K. Damle et al., Europhys. Lett. 36, 7 (1996).

[17] M. Machida, S. Yamada, Y. Ohashi, and H. Matsumoto, Phys. Rev. A 74, 053621 (2006). 